\title{
Study for Determination of Suitable Pre treatment Combination and Dehydration Temperature for Broccoli Florets
}

\author{
Ankan Das* and R. S. Dhua
}

Department of Post Harvest Technology of Horticultural Crops, Faculty of Horticulture, Bidhan Chandra Krishi Viswavidyalaya, Nadia West Bengal, 741252, India

Department of Horticulture, Institute of Agricultural Science, University of Calcutta, 51/2 Hazra Road, Kolkata 700019, India

*Corresponding author

\section{A B S T R A C T}

Keywords

Broccoli,

Blanching,

Chemicals,

Dehydration,

Packaging

\section{Article Info}

Accepted:

07 September 2019

Available Online:

10 October 2019
High moisture content of broccoli limits its post harvest longevity. So in order to make it available for a longer period the vegetable is needed to be preserved. For this, the present study was undertaken where dehydration was chosen as a mode of preservation and the experiment was aimed to establish suitable pre drying treatment combination and dehydration temperature for broccoli florets. Hot water blanching and chemicals like calcium chloride, citric acid, sodium metabisulfite and potassium metabisulfite were used for pre drying treatments. Three different temperatures of $50^{\circ} \mathrm{C}, 55^{\circ} \mathrm{C}$ and $60^{\circ} \mathrm{C}$ were employed for dehydration. The dehydrated broccoli florets were pre packed and stored at ambient condition. Storage studies for different physical and biochemical parameters were carried at proper intervals of storage. The work revealed that the pre drying treatment combination of initial immersion of $0.2 \%$ of calcium chloride followed by 4 minutes of hot water blanching and final immersion of $0.1 \%$ of sodium metabisulfite followed by dehydration at $55^{\circ} \mathrm{C}$ was most effective in maintaining the various physical and biochemical attributes throughout the storage period.

\section{Introduction}

The vegetable broccoli possesses very important group of chemicals which helps in prevention of a number of diseases. According to Gullett et al., (2010) sulforaphane and some other phytochemicals present in broccoli like indole-3-carbinol and brassinin are very much useful against cancer.

Apart from having anti cancerous properties broccoli is one of the very few vegetable that is also very effective against diabetes. The work carried out by Platel and Srinivasan, 
(1997) showed broccoli have beneficial influence against diabetes in humans as well as in experimental animals. Though broccoli contains numerous functional properties, but the high moisture content present in the vegetable restricts its post harvest life to a limited period. So in order to increase the post harvest utility, it is needed to preserve the vegetable for a longer period of time. For this dehydration can be done where broccoli, by reducing its moisture content can be successfully preserved for an extended span. Dehydration helps in reducing the moisture content to a great extent as a result of which the total volume gets minimized reducing the transformational cost, the chances of microbial contamination becomes less and ultimately the shelf life is prolonged (Kordylas, 1990).

Prior to dehydration of vegetables, various pretreatments are needed which helps in yielding final products of sound quality (Kingsly et al., 2007). Enzymes like peroxidase and lipoxygenase which are present in fresh vegetables causes undesirable chemical reactions that leads to change of colour from green to brown (Vamos-Vigyzao, 1995; McEvily et al., 1992).

So in order to overcome the issue apart for giving pretreatments blanching is also required to be done before dehydration as it helps in inactivation the enzymatic action as a result of which the colour and taste of the commodity is improved.

Furthermore this process of blanching helps in alleviating the internal elastic properties which facilitates the dehydration procedure (Kunzek et al., 1999; Munyaka et al., 2010; Waldron et al., 2003). Therefore the present study was carried to establish a pretreatment combination for successful dehydration and also to determine a suitable temperature in which the broccoli florets can be properly dehydrated.

\section{Materials and Methods}

The study was taken during the year 20152016 in the Department of Post Harvest Technology of Horticultural Crops, Faculty of Horticulture, Bidhan Chandra Krishi Viswavidyalaya, Nadia West Bengal. Broccoli variety Galaxy ( $F_{1}$ hybrid) was collected from a farmer field located at Nadia and North 24 Parganas districts of West Bengal. In the laboratory the broccoli heads after proper washing was cut into small florets and subjected to the different treatment combination as follows (immersion in chemical solution for 10 minutes + hot water blanching for 4 minutes + immersion in chemical solution for 10 minutes). The way of application of the treatments and some chemicals used in the study are similar to the works of Das and Dhua (2019) and Ngangom et al., (2019).

$\mathbf{T}_{\mathbf{1}}-$ Citric acid $0.2 \%+4 \mathrm{~min}$ blanching + water,

$\mathbf{T}_{\mathbf{2}}-$ Citric acid $0.2 \%+4$ min blanching + potassium metabisulphite $0.1 \%$,

$\mathbf{T}_{\mathbf{3}}-$ Citric acid $0.2 \%+4 \mathrm{~min}$ blanching + Sodium metabisulfite $0.1 \%$,

$\mathbf{T}_{\mathbf{4}}$ Calcium chloride $0.2 \%+4$ min blanching + water,

$\mathbf{T}_{\mathbf{5}}-$ Calcium chloride $0.2 \%+4 \mathrm{~min}$ blanching + potassium metabisulphite $0.1 \%$,

$\mathbf{T}_{\mathbf{6}}-$ Calcium chloride $0.2 \%+4 \mathrm{~min}$ blanching + Sodium metabisulfite $0.1 \%$,

$\mathbf{T}_{7}-$ water +4 min blanching + water

\section{Design of experiment}

Two Factorial Completely Randomized Design (Sheoran et al., 1998). 


\section{Replication- 3}

After that, drying was undertaken at the temperatures of $50^{\circ} \mathrm{C}, \quad 55^{\circ} \mathrm{C}$ and $60^{\circ} \mathrm{C}$. Thereafter dehydrated florets were pre packed and stored in ambient situation. Analysis of different attributes viz. matter content dry weight basis (Shipley and $\mathrm{Vu}, 2002$ ), moisture content of dehydrated produce (A.O.A.C, 2000), rehydration ratio (A.O.A.C, 2000), total chlorophyll (Ranganna, 2003), total phenols (Singleton et al., 1999), flavanoids (Zhishen et al., 1999), antioxidant activity (Brand-Williams et al., 1995) and fungal estimation (Allen, 1953) were carried on 0, 30, 45 and 60 days of storage.

\section{Results and Discussion}

All the treatments under the three temperatures viz. $50^{\circ} \mathrm{C}, \quad 55^{\circ} \mathrm{C}$ and $60^{\circ} \mathrm{C}$ showed maximum decrease in the moisture content (dry wt. basis) during the initial periods of dehydration (Fig. 1, 2 and 3). But later on the reduction of content among the treatments stabilized with ongoing time period during dehydration. For $50^{\circ} \mathrm{C}$ a time span of 720 minutes was required to stabilize the moisture content (dry wt. basis) for all the treatments and after which no further decrease in the value was observed. For the temperature of $55^{\circ} \mathrm{C}$ the time period required for all the treatments for stabilization was observed at 570 minutes. The temperature of $60^{\circ} \mathrm{C}$ required a lesser time of 510 minutes to bring down the moisture content (dry wt. basis) for all the treatments.

During the period of storage the moisture content for all the treatments dehydrated at different temperatures viz. $50^{\circ} \mathrm{C} 55^{\circ} \mathrm{C}$ and $60^{\circ} \mathrm{C}$ increased (Table 1). Treatments dehydrated at $50^{\circ} \mathrm{C}$ showed maximum increase in the moisture levels throughout the period of storage. Treatments dehydrated at $55^{\circ} \mathrm{C}$ and $60^{\circ} \mathrm{C}$ maintained a steady rate of moisture gain during the storage period, with lowest levels of moisture content was recorded for the treatments dehydrated at $60^{\circ} \mathrm{C}$ at the end of the storage. Among the different treatments the broccoli florets in which initial immersion with $0.2 \%$ of calcium chloride followed by 4 minutes of hot water blanching and final immersion with $0.1 \%$ of sodium metabisulfite was done, showed the least uptake of moisture.

The maximum rehydration ratio of 7.25 at the 0 days of storage was seen for treatments dehydrated at $50^{\circ} \mathrm{C}$ followed by 5.68 for treatments dehydrated at $55^{\circ} \mathrm{C}$ and 4.94 for treatments dehydrated at $60^{\circ} \mathrm{C}$ respectively (Table 2). The rehydration ratio throughout the storage period for different treatments dehydrated at each temperature decreased. At the end of 60 days of storage treatments dehydrated at $50^{\circ} \mathrm{C}$ recorder the maximum rehydration ratio of 6.23 in broccoli florets where initial immersion with $0.2 \%$ of calcium chloride, 4 minutes of hot water blanching and final immersion with $0.1 \%$ of sodium metabisulfite was done.

After 30 days of storage treatments dehydrated at $50^{\circ} \mathrm{C}$ showed the maximum concentration of total chlorophyll followed by treatments which were dehydrated at $55^{\circ} \mathrm{C}$ and $60^{\circ} \mathrm{C}$ respectively (Table 3). However at 45 days of storage, treatments dehydrated at $55^{\circ} \mathrm{C}$ showed similar concentrations of total chlorophyll reatinment as compared to treatments dehydrated at $50^{\circ} \mathrm{C}$ whereas concentration for chlorophyll for different treatments dehydrated at $60^{\circ} \mathrm{C}$ was at the lower side. At the end of storage at 60 days, considerable loss in the total chlorophyll content was seen for all the treatments dehydrated at $50^{\circ} \mathrm{C}$ and $60^{\circ} \mathrm{C}$. Treatments dehydrated at temperature of $55^{\circ} \mathrm{C}$ recorded the maximum concentration of chlorophyll at 60 days of storage. Treatment of broccoli florets where initial immersion with $0.2 \%$ of calcium chloride, 4 minutes of 
hot water blanching and final immersion with $0.1 \%$ of sodium metabisulfite which were dehydrated at a temperature of $55^{\circ} \mathrm{C}$ maintained a significant higher level of total chlorophyll concentration throughout the storage period

Biochemical parameters like phenols, flavanoids and antioxidant levels (\% inhibition of DPPH) were highest for all treatments at 0 days of storage for dehydration temperature of $50^{\circ} \mathrm{C}$ followed by dehydration temperature of $55^{\circ} \mathrm{C}$ and dehydration temperature of $60^{\circ} \mathrm{C}$ (Table 4, 5, 6). But later during the storage period the concentration of phenols, flavanoids and antioxidant levels (\% inhibition of $\mathrm{DPPH}$ ) decreased for all the treatments dehydrated at temperature of $50^{\circ} \mathrm{C}$ and $60^{\circ} \mathrm{C}$. Treatments dehydrated at $55^{\circ} \mathrm{C}$ showed the maximum possession of phenols, flavanoids and antioxidant levels throughout the period of storage. Dehydrated at the temperature $55^{\circ} \mathrm{C} / \mathrm{B}_{2}$ after 60 days of storage, the broccoli florets were initial immersion with $0.2 \%$ of calcium chloride, 4 minutes of hot water blanching and final immersion with $0.1 \%$ of sodium metabisulfite showed the maximum levels of phenols, flavanoids and antioxidant activity

The fungal count for both unicellular and filamentous type were lowest at the initial day of storage for treatments dehydrated at $60^{\circ} \mathrm{C}$ (unicellular fungi: $1.33 \times 10^{2} \mathrm{cfu} / \mathrm{g}$, filamentous fungi: $0.66 \times 10^{2} \mathrm{cfu} / \mathrm{g}$ ) followed by treatments dehydrated at $55^{\circ} \mathrm{C}$ and $50^{\circ} \mathrm{C}$ (Table 7 and 8).

The microbial population for the treatments dehydrated at temperature of $50^{\circ} \mathrm{C}, 55^{\circ} \mathrm{C}$ and $60^{\circ} \mathrm{C}$ increased during the storage period. Treatments dehydrated at the temperature of $50^{\circ} \mathrm{C}$ showed the highest levels of fungal population. However treatments dehydrated at temperature of $55^{\circ} \mathrm{C}$ and $60^{\circ} \mathrm{C}$ maintained a lower rate of fungal infestation throughout the storage period of 60 days. At the end of the experiment broccoli florets where initial immersion with $0.2 \%$ of calcium chloride, 4 minutes of hot water blanching and final immersion with $0.1 \%$ of sodium metabisulfite was done and dehydration was carried at a temperature of $60^{\circ} \mathrm{C}$ showed the lowest fungal population of $2 \times 10^{2} \mathrm{cfu} / \mathrm{g}$ (unicellular type) and $0.67 \times \quad 10^{2} \mathrm{cfu} / \mathrm{g}$ (filamentous type) respectively.

For fresh vegetable various enzymes like lipoxygenase and peroxidase are responsible for development of brown colour due to enzymatic reaction and also results in the occurrence of unpleasant odour (VamosVigyzao, 1995; McEvily et al., 1992).

These problems are lessened by the help of dehydration as it helps in reduction of free water content which in turn reduces the microbial affinity and ultimately increases the post harvest life (Hatamipour et al., 2007). Before dehydration the broccoli florets were blanched with hot water which facilitates the drying and ensures proper shrinkage (Kunzek et al., 1999; Munyaka et al., 2010; Waldron et al., 2003) and various pre drying treatments were given.

Previous studies have reported that treating the cut tissues of the vegetable helps in reducing the rate of respiration and escalates the healing process (Picchioni, 1994) and also the tissue firmness is elevated (Rosen and Kader, 1989; Izumi and Watada, 1994).

In the experiment the effectiveness of chemicals like calcium chloride and sodium meta bisulphite as pre drying treatments were observed. The findings were at par to that of Owureku et al., (2014) were tomato fruits pretreated with sodium metabisulfite were uniformly dehydrated with least degradation of chlorophyll and maximum rehydration ration than the other treatments. 
Table.1 Moisture content (\%) of dehydrated broccoli florets during the storage intervals

\begin{tabular}{|c|c|c|c|c|}
\hline O DAS & $50^{0} \mathrm{C} / \mathrm{B}_{1}$ & $55^{0} \mathrm{C} / \mathrm{B}_{2}$ & $60^{0} \mathrm{C} / \mathrm{B}_{3}$ & Mean A \\
\hline $\mathbf{A}_{1}-\mathbf{A}_{7}$ & 7.14 & 5.66 & 5.00 & - \\
\hline Mean B & - & - & - & \\
\hline 30 DAS & $\mathbf{B}_{1}$ & $\mathbf{B}_{2}$ & $\mathbf{B}_{3}$ & Mean A \\
\hline $\mathbf{T}_{1} / \mathbf{A}_{1}$ & 7.66 & 6.22 & 5.39 & 6.42 \\
\hline $\mathbf{T}_{2} / \mathbf{A}_{2}$ & 7.66 & 6.16 & 5.38 & 6.40 \\
\hline $\mathbf{T}_{\mathbf{3}} / \mathbf{A}_{\mathbf{3}}$ & 7.26 & 5.92 & 5.28 & 6.15 \\
\hline $\mathbf{T}_{4} / \mathbf{A}_{4}$ & 7.91 & 6.52 & 5.62 & 6.68 \\
\hline $\mathbf{T}_{5} / \mathbf{A}_{5}$ & 7.45 & 5.96 & 5.35 & 6.25 \\
\hline $\mathbf{T}_{6} / \mathbf{A}_{6}$ & 7.24 & 5.87 & 5.23 & 6.11 \\
\hline $\mathbf{T}_{7} / \mathbf{A}_{7}$ & 7.98 & 6.58 & 5.65 & 6.73 \\
\hline \multirow[t]{5}{*}{ Mean B } & 7.60 & 6.18 & 5.41 & \\
\hline & Factors & C.D. & SE(d) & SE(m) \\
\hline & Factor(A) & 0.013 & 0.006 & 0.005 \\
\hline & Factor(B) & 0.009 & 0.004 & 0.003 \\
\hline & Factor $(\mathrm{AxB})$ & 0.023 & 0.011 & 0.008 \\
\hline 45 DAS & $50^{0} \mathrm{C} / \mathrm{B}_{1}$ & $55^{0} \mathrm{C} / \mathrm{B}_{2}$ & $60^{0} \mathrm{C} / \mathrm{B}_{3}$ & Mean A \\
\hline $\mathbf{T}_{1} / \mathbf{A}_{1}$ & 8.38 & 6.46 & 6.41 & 7.08 \\
\hline $\mathbf{T}_{2} / \mathbf{A}_{2}$ & 8.32 & 6.39 & 6.28 & 7.00 \\
\hline$T_{3} / A_{3}$ & 8.22 & 6.22 & 5.98 & 6.81 \\
\hline $\mathbf{T}_{4} / \mathbf{A}_{4}$ & 8.97 & 6.52 & 6.45 & 7.31 \\
\hline $\mathbf{T}_{5} / \mathbf{A}_{5}$ & 8.25 & 6.35 & 6.23 & 6.95 \\
\hline $\mathbf{T}_{6} / \mathbf{A}_{6}$ & 8.14 & 6.16 & 5.92 & 6.74 \\
\hline $\mathbf{T}_{7} / \mathbf{A}_{7}$ & 9.20 & 6.78 & 6.45 & 7.47 \\
\hline \multirow[t]{5}{*}{ Mean B } & 8.50 & 6.41 & 6.25 & \\
\hline & Factors & C.D. & SE(d) & SE(m) \\
\hline & Factor(A) & 0.020 & 0.010 & 0.007 \\
\hline & Factor(B) & 0.013 & 0.007 & 0.005 \\
\hline & Factor $(\mathrm{AxB})$ & 0.035 & 0.017 & 0.012 \\
\hline 60 DAS & $50^{0} \mathrm{C} / \mathrm{B}_{1}$ & $55^{0} \mathrm{C} / \mathrm{B}_{2}$ & $60^{0} \mathrm{C} / \mathrm{B}_{3}$ & Mean A \\
\hline $\mathbf{T}_{1} / \mathbf{A}_{1}$ & 12.23 & 7.69 & 7.15 & 9.02 \\
\hline $\mathbf{T}_{2} / \mathbf{A}_{2}$ & 11.63 & 7.45 & 6.75 & 8.61 \\
\hline $\mathbf{T}_{3} / \mathbf{A}_{3}$ & 8.32 & 6.72 & 6.32 & 7.12 \\
\hline $\mathbf{T}_{4} / \mathbf{A}_{4}$ & 12.90 & 8.23 & 7.24 & 9.46 \\
\hline $\mathbf{T}_{5} / \mathbf{A}_{5}$ & 11.13 & 6.97 & 6.44 & 8.18 \\
\hline$T_{6} / A_{6}$ & 8.26 & 6.64 & 6.28 & 7.06 \\
\hline $\mathbf{T}_{7} / \mathbf{A}_{7}$ & 13.29 & 8.34 & 7.29 & 9.64 \\
\hline \multirow[t]{5}{*}{ Mean B } & 11.11 & 7.43 & 6.78 & \\
\hline & Factors & C.D. & SE(d) & SE(m) \\
\hline & Factor(A) & 0.035 & 0.018 & 0.012 \\
\hline & Factor(B) & 0.023 & 0.011 & 0.008 \\
\hline & Factor $(\mathrm{AxB})$ & 0.061 & 0.030 & 0.021 \\
\hline
\end{tabular}

A(1-7): Treatments $\left\{\mathbf{A 1}\left(\mathrm{T}_{1}\right)-\right.$ Citric acid $0.2 \%+4$ min blanching + water, $\mathbf{A 2}\left(\mathrm{T}_{2}\right)-$ Citric acid $0.2 \%+4 \mathrm{~min}$ blanching $+\mathrm{K}_{2} \mathrm{~S}_{2} \mathrm{O}_{5} 0.1 \%, \mathbf{A 3}\left(\mathrm{T}_{3}\right)-$ Citric acid $0.2 \%+4$ min blanching $+\mathrm{Na}_{2} \mathrm{~S}_{2} \mathrm{O}_{5} 0.1 \%, \mathrm{A4}_{4}\left(\mathrm{~T}_{4}\right)-\mathrm{CaCl}_{2} 0.2 \%+4$ min blanching + water, $\mathbf{A 5}\left(\mathrm{T}_{5}\right)-\mathrm{CaCl}_{2} 0.2 \%+4$ min blanching $+\mathrm{K}_{2} \mathrm{~S}_{2} \mathrm{O}_{5} 0.1 \%$, $\mathbf{A 6}\left(\mathrm{T}_{6}\right)-\mathrm{CaCl}_{2} 0.2 \%+4$ min blanching $+\mathrm{Na}_{2} \mathrm{~S}_{2} \mathrm{O}_{5} 0.1 \%, \mathbf{A 7}\left(\mathrm{T}_{7}\right)-$ water +4 min blanching + water $\}, \mathrm{B}(1-3)$ : Temperatures $\left(\mathbf{B} 1-50^{\circ} \mathrm{C}\right.$, B2$55^{\circ} \mathrm{C}, \mathrm{B3}-60^{\circ} \mathrm{C}$ ) 
Table.2 Rehydration ratio of dehydrated broccoli florets during the storage intervals

\begin{tabular}{|c|c|c|c|c|}
\hline 0 DAS & $50^{0} \mathrm{C} / \mathrm{B}_{1}$ & $55^{0} \mathrm{C} / \mathrm{B}_{2}$ & $60^{0} \mathrm{C} / \mathrm{B}_{3}$ & Mean A \\
\hline $\mathbf{A}_{1}-\mathbf{A}_{7}$ & 7.25 & 5.68 & 4.94 & - \\
\hline Mean B & - & - & - & \\
\hline 30 DAS & $\mathbf{B}_{1}$ & $\mathbf{B}_{2}$ & $\mathbf{B}_{3}$ & Mean A \\
\hline $\mathbf{T}_{1} / \mathbf{A}_{1}$ & 6.88 & 5.34 & 4.64 & 5.62 \\
\hline $\mathbf{T}_{2} / \mathbf{A}_{2}$ & 6.92 & 5.52 & 4.81 & 5.75 \\
\hline $\mathbf{T}_{\mathbf{3}} / \mathbf{A}_{\mathbf{3}}$ & 6.96 & 5.54 & 4.85 & 5.78 \\
\hline $\mathbf{T}_{4} / \mathbf{A}_{4}$ & 6.56 & 5.32 & 4.53 & 5.47 \\
\hline $\mathbf{T}_{5} / \mathbf{A}_{5}$ & 6.96 & 5.52 & 4.81 & 5.77 \\
\hline$T_{6} / A_{6}$ & 7.05 & 5.62 & 4.87 & 5.85 \\
\hline $\mathbf{T}_{7} / \mathbf{A}_{7}$ & 6.56 & 5.26 & 5.26 & 5.69 \\
\hline \multirow[t]{5}{*}{ Mean B } & 6.84 & 5.45 & 4.83 & \\
\hline & Factors & C.D. & SE(d) & $\mathbf{S E}(\mathbf{m})$ \\
\hline & Factor(A) & 0.013 & 0.006 & 0.005 \\
\hline & Factor(B) & 0.009 & 0.004 & 0.003 \\
\hline & Factor $(\mathrm{AxB})$ & 0.023 & 0.011 & 0.008 \\
\hline 45 DAS & $50^{0} \mathrm{C} / \mathrm{B}_{1}$ & $55^{0} \mathrm{C} / \mathrm{B}_{2}$ & $60^{0} \mathrm{C} / \mathrm{B}_{3}$ & Mean A \\
\hline $\mathbf{T}_{1} / \mathbf{A}_{1}$ & 5.97 & 5.16 & 4.14 & 5.09 \\
\hline $\mathbf{T}_{2} / \mathbf{A}_{2}$ & 6.13 & 5.22 & 4.27 & 5.21 \\
\hline $\mathbf{T}_{3} / \mathbf{A}_{3}$ & 6.91 & 5.33 & 4.33 & 5.52 \\
\hline $\mathbf{T}_{4} / \mathbf{A}_{4}$ & 5.94 & 4.96 & 3.98 & 4.96 \\
\hline $\mathbf{T}_{5} / \mathbf{A}_{5}$ & 6.62 & 5.41 & 4.31 & 5.44 \\
\hline $\mathbf{T}_{6} / \mathbf{A}_{6}$ & 6.83 & 5.46 & 4.38 & 5.56 \\
\hline $\mathbf{T}_{7} / \mathbf{A}_{7}$ & 5.91 & 4.95 & 3.93 & 4.93 \\
\hline \multirow[t]{5}{*}{ Mean B } & 6.33 & 5.21 & 4.19 & \\
\hline & Factors & C.D. & SE(d) & $\mathrm{SE}(\mathrm{m})$ \\
\hline & Factor(A) & 0.039 & 0.019 & 0.014 \\
\hline & Factor(B) & 0.026 & 0.013 & 0.009 \\
\hline & Factor $(\mathrm{AxB})$ & 0.068 & 0.034 & 0.024 \\
\hline 60 DAS & $50^{0} \mathrm{C} / \mathrm{B}_{1}$ & $55^{0} \mathrm{C} / \mathrm{B}_{2}$ & $60^{\circ} \mathrm{C} / \mathrm{B}_{3}$ & Mean A \\
\hline $\mathbf{T}_{1} / \mathbf{A}_{1}$ & 5.35 & 4.33 & 3.79 & 4.49 \\
\hline $\mathbf{T}_{2} / \mathbf{A}_{2}$ & 5.71 & 4.64 & 3.86 & 4.74 \\
\hline $\mathbf{T}_{\mathbf{3}} / \mathbf{A}_{\mathbf{3}}$ & 6.03 & 4.81 & 4.18 & 5.01 \\
\hline $\mathbf{T}_{4} / \mathbf{A}_{4}$ & 5.13 & 4.31 & 3.66 & 4.37 \\
\hline $\mathbf{T}_{5} / \mathbf{A}_{5}$ & 5.74 & 4.81 & 4.02 & 4.86 \\
\hline $\mathrm{T}_{6} / \mathrm{A}_{6}$ & 6.23 & 4.84 & 4.18 & 5.08 \\
\hline $\mathbf{T}_{7} / \mathbf{A}_{7}$ & 5.05 & 4.31 & 3.64 & 4.33 \\
\hline \multirow[t]{5}{*}{ Mean B } & 5.61 & 4.58 & 3.90 & \\
\hline & Factors & C.D. & SE(d) & $\mathbf{S E}(\mathbf{m})$ \\
\hline & Factor(A) & 0.014 & 0.007 & 0.005 \\
\hline & Factor(B) & 0.009 & 0.004 & 0.003 \\
\hline & Factor $(\mathrm{AxB})$ & 0.024 & 0.012 & 0.008 \\
\hline
\end{tabular}

A(1-7): Treatments $\left\{\mathbf{A 1}\left(\mathrm{T}_{1}\right)-\right.$ Citric acid $0.2 \%+4$ min blanching + water, $\mathbf{A 2}\left(\mathrm{T}_{2}\right)-$ Citric acid $0.2 \%+4 \mathrm{~min}$ blanching $+\mathrm{K}_{2} \mathrm{~S}_{2} \mathrm{O}_{5} 0.1 \%, \mathbf{A 3}\left(\mathrm{T}_{3}\right)-$ Citric acid $0.2 \%+4$ min blanching $+\mathrm{Na}_{2} \mathrm{~S}_{2} \mathrm{O}_{5} 0.1 \%, \mathbf{A 4}\left(\mathrm{T}_{4}\right)-\mathrm{CaCl}_{2} 0.2 \%+4$ min blanching + water, $\mathbf{A 5}\left(\mathrm{T}_{5}\right)-\mathrm{CaCl}_{2} 0.2 \%+4$ min blanching $+\mathrm{K}_{2} \mathrm{~S}_{2} \mathrm{O}_{5} 0.1 \%, \mathbf{A 6}\left(\mathrm{T}_{6}\right)-\mathrm{CaCl}_{2} 0.2 \%+4 \min$ blanching $+\mathrm{Na}_{2} \mathrm{~S}_{2} \mathrm{O}_{5} 0.1 \%, \mathbf{A 7}\left(\mathrm{T}_{7}\right)-$ water +4 min blanching + water $\}, \mathrm{B}(1-3)$ : Temperatures $\left(\mathbf{B 1}-50^{\circ} \mathrm{C}, \mathbf{B 2}\right.$ $\left.55^{\circ} \mathrm{C}, \mathrm{B3}-60^{\circ} \mathrm{C}\right)$ 
Table.3 Total chlorophyll (mg/g) of dehydrated broccoli florets during the storage intervals

\begin{tabular}{|c|c|c|c|c|}
\hline 0 DAS & $50^{0} \mathrm{C} / \mathrm{B}_{1}$ & $55^{0} \mathrm{C} / \mathrm{B}_{2}$ & $60^{0} \mathrm{C} / \mathrm{B}_{3}$ & Mean A \\
\hline $\mathbf{A}_{1}-\mathbf{A}_{7}$ & 9.70 & 9.13 & 8.26 & - \\
\hline Mean B & - & - & - & \\
\hline 30 DAS & $\mathbf{B}_{1}$ & $\mathbf{B}_{2}$ & $\mathbf{B}_{3}$ & Mean A \\
\hline$T_{1} / A_{1}$ & 7.07 & 4.63 & 4.47 & 5.39 \\
\hline $\mathbf{T}_{2} / \mathbf{A}_{2}$ & 7.40 & 6.07 & 5.17 & 6.21 \\
\hline $\mathbf{T}_{\mathbf{3}} / \mathbf{A}_{\mathbf{3}}$ & 8.57 & 7.27 & 6.73 & 7.52 \\
\hline $\mathbf{T}_{4} / \mathbf{A}_{4}$ & 6.43 & 4.27 & 3.60 & 4.77 \\
\hline $\mathbf{T}_{5} / \mathbf{A}_{5}$ & 8.07 & 6.50 & 5.83 & 6.80 \\
\hline $\mathbf{T}_{6} / \mathbf{A}_{6}$ & 8.87 & 7.50 & 7.03 & 7.80 \\
\hline $\mathbf{T}_{7} / \mathbf{A}_{7}$ & 6.17 & 3.40 & 3.07 & 4.21 \\
\hline \multirow[t]{5}{*}{ Mean B } & 7.51 & 5.66 & 5.13 & \\
\hline & Factors & C.D. & SE(d) & $\mathbf{S E}(\mathbf{m})$ \\
\hline & Factor(A) & 0.129 & 0.064 & 0.045 \\
\hline & Factor(B) & 0.084 & 0.042 & 0.029 \\
\hline & Factor AxB & 0.223 & 0.11 & 0.078 \\
\hline 45 DAS & $50^{0} \mathrm{C} / \mathbf{B}_{1}$ & $55^{0} \mathrm{C} / \mathrm{B}_{2}$ & $60^{0} \mathrm{C} / \mathrm{B}_{3}$ & Mean A \\
\hline $\mathbf{T}_{1} / \mathbf{A}_{1}$ & 4.40 & 3.67 & 3.47 & 3.84 \\
\hline $\mathbf{T}_{2} / \mathbf{A}_{2}$ & 6.10 & 4.33 & 4.03 & 4.82 \\
\hline $\mathbf{T}_{\mathbf{3}} / \mathbf{A}_{\mathbf{3}}$ & 7.77 & 6.10 & 5.30 & 6.39 \\
\hline $\mathbf{T}_{4} / \mathbf{A}_{4}$ & 3.83 & 3.27 & 2.80 & 3.30 \\
\hline $\mathbf{T}_{5} / \mathbf{A}_{5}$ & 6.83 & 5.67 & 5.03 & 5.84 \\
\hline $\mathbf{T}_{6} / \mathbf{A}_{6}$ & 8.30 & 6.80 & 6.10 & 7.07 \\
\hline $\mathbf{T}_{7} / \mathbf{A}_{7}$ & 3.13 & 3.00 & 2.20 & 2.78 \\
\hline \multirow[t]{5}{*}{ Mean B } & 5.77 & 4.69 & 4.13 & \\
\hline & Factors & C.D. & $\mathrm{SE}(\mathrm{d})$ & SE(m) \\
\hline & Factor(A) & 0.158 & 0.078 & 0.055 \\
\hline & Factor(B) & 0.104 & 0.051 & 0.036 \\
\hline & Factor AxB & 0.274 & 0.135 & 0.096 \\
\hline 60 DAS & $50^{0} \mathrm{C} / \mathrm{B}_{1}$ & $55^{\circ} \mathrm{C} / \mathrm{B}_{2}$ & $60^{\circ} \mathrm{C} / \mathrm{B}_{3}$ & Mean A \\
\hline $\mathbf{T}_{1} / \mathbf{A}_{1}$ & 2.70 & 2.43 & 2.73 & 2.62 \\
\hline $\mathbf{T}_{2} / \mathbf{A}_{2}$ & 3.40 & 3.20 & 3.17 & 3.26 \\
\hline $\mathbf{T}_{3} / \mathbf{A}_{3}$ & 5.43 & 5.77 & 5.07 & 5.42 \\
\hline $\mathbf{T}_{4} / \mathbf{A}_{4}$ & 2.13 & 2.93 & 1.80 & 2.29 \\
\hline $\mathbf{T}_{5} / \mathbf{A}_{5}$ & 4.50 & 4.73 & 4.17 & 4.47 \\
\hline $\mathbf{T}_{6} / \mathrm{A}_{6}$ & 6.13 & 6.27 & 5.63 & 6.01 \\
\hline $\mathbf{T}_{7} / \mathbf{A}_{7}$ & 1.63 & 1.83 & 1.13 & 1.53 \\
\hline \multirow[t]{5}{*}{ Mean B } & 3.71 & 3.88 & 3.39 & \\
\hline & Factors & C.D. & SE(d) & SE(m) \\
\hline & Factor(A) & 0.132 & 0.065 & 0.046 \\
\hline & Factor(B) & 0.087 & 0.043 & 0.03 \\
\hline & Factor AxB & 0.229 & 0.113 & 0.08 \\
\hline
\end{tabular}

$\mathbf{A}(1-7)$ : Treatments $\left\{\mathbf{A 1}\left(\mathrm{T}_{1}\right)-\right.$ Citric acid $0.2 \%+4$ min blanching + water, $\mathbf{A 2}\left(\mathrm{T}_{2}\right)-$ Citric acid $0.2 \%+4 \mathrm{~min}$ blanching $+\mathrm{K}_{2} \mathrm{~S}_{2} \mathrm{O}_{5} 0.1 \%$, A3 $\left(\mathrm{T}_{3}\right)-$ Citric acid $0.2 \%+4$ min blanching $+\mathrm{Na}_{2} \mathrm{~S}_{2} \mathrm{O}_{5} 0.1 \%, \mathbf{A 4}\left(\mathrm{T}_{4}\right)-\mathrm{CaCl}_{2} 0.2 \%+4$ min blanching + water, $\mathbf{A 5}\left(\mathrm{T}_{5}\right)-\mathrm{CaCl}_{2} 0.2 \%+4$ min blanching $+\mathrm{K}_{2} \mathrm{~S}_{2} \mathrm{O}_{5} 0.1 \%, \mathbf{A 6}\left(\mathrm{T}_{6}\right)-\mathrm{CaCl}_{2} 0.2 \%+4 \min$ blanching $+\mathrm{Na}_{2} \mathrm{~S}_{2} \mathrm{O}_{5} 0.1 \%, \mathbf{A} 7\left(\mathrm{~T}_{7}\right)-$ water +4 min blanching + water $\}, \mathrm{B}(1-3)$ : Temperatures $\left(\mathbf{B} 1-50^{\circ} \mathrm{C}, \mathbf{B} 2-\right.$ $\left.55^{\circ} \mathrm{C}, \mathbf{B 3}-60^{\circ} \mathrm{C}\right)$ 
Table.4 Total content of phenols (mg GAE/g) of dehydrated broccoli florets during the storage intervals

\begin{tabular}{|c|c|c|c|c|}
\hline 0 DAS & $50^{0} \mathrm{C} / \mathrm{B}_{1}$ & $55^{0} \mathrm{C} / \mathrm{B}_{2}$ & $60^{0} \mathrm{C} / \mathrm{B}_{3}$ & Mean A \\
\hline $\mathbf{A}_{1}-\mathbf{A}_{7}$ & 5.83 & 5.24 & 4.73 & - \\
\hline Mean B & - & - & - & \\
\hline 30 days & $\mathbf{B}_{1}$ & $\mathbf{B}_{2}$ & $\mathbf{B}_{3}$ & Mean A \\
\hline $\mathbf{T}_{1} / \mathbf{A}_{1}$ & 4.92 & 4.08 & 3.34 & 4.11 \\
\hline $\mathbf{T}_{2} / \mathbf{A}_{2}$ & 5.05 & 4.23 & 3.64 & 4.31 \\
\hline $\mathbf{T}_{\mathbf{3}} / \mathbf{A}_{3}$ & 5.36 & 4.74 & 4.14 & 4.75 \\
\hline $\mathbf{T}_{4} / \mathbf{A}_{4}$ & 4.85 & 4.01 & 3.08 & 3.98 \\
\hline $\mathbf{T}_{5} / \mathbf{A}_{5}$ & 5.25 & 4.55 & 3.77 & 4.52 \\
\hline $\mathrm{T}_{6} / \mathrm{A}_{6}$ & 5.55 & 4.84 & 4.45 & 4.95 \\
\hline $\mathbf{T}_{7} / \mathbf{A}_{7}$ & 4.63 & 3.82 & 2.85 & 3.76 \\
\hline \multirow[t]{5}{*}{ Mean B } & 5.09 & 4.32 & 3.61 & \\
\hline & Factors & C.D. & SE(d) & $\mathbf{S E}(\mathbf{m})$ \\
\hline & Factor(A) & 0.024 & 0.012 & 0.008 \\
\hline & Factor(B) & 0.015 & 0.008 & 0.005 \\
\hline & Factor(A X B) & 0.041 & 0.02 & 0.014 \\
\hline 45 days & $50^{0} \mathrm{C} / \mathrm{B}_{1}$ & $55^{0} \mathrm{C} / \mathrm{B}_{2}$ & $60^{0} \mathrm{C} / \mathrm{B}_{3}$ & Mean A \\
\hline $\mathbf{T}_{1} / \mathbf{A}_{1}$ & 3.05 & 2.96 & 2.65 & 2.88 \\
\hline $\mathbf{T}_{2} / \mathbf{A}_{2}$ & 3.57 & 3.52 & 3.26 & 3.45 \\
\hline $\mathbf{T}_{3} / \mathbf{A}_{3}$ & 4.35 & 4.08 & 3.76 & 4.06 \\
\hline $\mathbf{T}_{4} / \mathbf{A}_{4}$ & 2.65 & 2.52 & 2.24 & 2.47 \\
\hline $\mathbf{T}_{5} / \mathbf{A}_{5}$ & 3.92 & 3.82 & 3.61 & 3.78 \\
\hline$T_{6} / A_{6}$ & 4.73 & 4.66 & 4.16 & 4.52 \\
\hline $\mathbf{T}_{7} / \mathbf{A}_{7}$ & 2.22 & 2.05 & 1.85 & 2.04 \\
\hline \multirow[t]{5}{*}{ Mean B } & 3.50 & 3.37 & 3.07 & \\
\hline & Factors & C.D. & SE(d) & $\mathbf{S E}(\mathbf{m})$ \\
\hline & Factor(A) & 0.026 & 0.013 & 0.009 \\
\hline & Factor(B) & 0.017 & 0.008 & 0.006 \\
\hline & Factor(A X B) & 0.045 & 0.022 & 0.016 \\
\hline 60 days & $50^{0} \mathrm{C} / \mathrm{B}_{1}$ & $55^{0} \mathrm{C} / \mathrm{B}_{2}$ & $60^{0} \mathrm{C} / \mathrm{B}_{3}$ & Mean A \\
\hline $\mathbf{T}_{1} / \mathbf{A}_{1}$ & 1.55 & 2.15 & 1.42 & 1.71 \\
\hline $\mathbf{T}_{2} / \mathbf{A}_{2}$ & 1.77 & 2.32 & 1.59 & 1.89 \\
\hline $\mathbf{T}_{\mathbf{3}} / \mathbf{A}_{\mathbf{3}}$ & 2.16 & 3.07 & 1.82 & 2.35 \\
\hline $\mathbf{T}_{4} / \mathbf{A}_{4}$ & 1.25 & 1.76 & 1.04 & 1.35 \\
\hline $\mathbf{T}_{5} / \mathbf{A}_{5}$ & 1.95 & 2.55 & 1.65 & 2.05 \\
\hline $\mathrm{T}_{6} / \mathrm{A}_{6}$ & 2.59 & 3.35 & 2.41 & 2.78 \\
\hline $\mathbf{T}_{7} / \mathbf{A}_{7}$ & 0.96 & 1.44 & 0.84 & 1.08 \\
\hline \multirow[t]{5}{*}{ Mean B } & 1.75 & 2.38 & 1.54 & \\
\hline & Factors & C.D. & SE(d) & $\mathbf{S E}(\mathbf{m})$ \\
\hline & Factor(A) & 0.026 & 0.013 & 0.009 \\
\hline & Factor(B) & 0.017 & 0.008 & 0.006 \\
\hline & Factor(A X B) & 0.045 & 0.022 & 0.016 \\
\hline
\end{tabular}

A(1-7): Treatments $\left\{\mathbf{A 1}\left(\mathrm{T}_{1}\right)-\right.$ Citric acid $0.2 \%+4$ min blanching + water, $\mathbf{A 2}\left(\mathrm{T}_{2}\right)-$ Citric acid $0.2 \%+4 \mathrm{~min}$ blanching $+\mathrm{K}_{2} \mathrm{~S}_{2} \mathrm{O}_{5} 0.1 \%, \mathbf{A 3}\left(\mathrm{T}_{3}\right)-$ Citric acid $0.2 \%+4$ min blanching $+\mathrm{Na}_{2} \mathrm{~S}_{2} \mathrm{O}_{5} 0.1 \%, \mathbf{A 4}\left(\mathrm{T}_{4}\right)-\mathrm{CaCl}_{2} 0.2 \%+4$ min blanching + water, $\mathbf{A 5}\left(\mathrm{T}_{5}\right)-\mathrm{CaCl}_{2} 0.2 \%+4$ min blanching $+\mathrm{K}_{2} \mathrm{~S}_{2} \mathrm{O}_{5} 0.1 \%, \mathbf{A 6}\left(\mathrm{T}_{6}\right)-\mathrm{CaCl}_{2} 0.2 \%+4 \min$ blanching $+\mathrm{Na}_{2} \mathrm{~S}_{2} \mathrm{O}_{5} 0.1 \%, \mathbf{A} 7\left(\mathrm{~T}_{7}\right)-$ water +4 min blanching + water $\}, \mathrm{B}(1-3)$ : Temperatures $\left(\mathbf{B} 1-50^{\circ} \mathrm{C}, \mathbf{B} 2-\right.$ $\left.55^{\circ} \mathrm{C}, \mathrm{B3}-60^{\circ} \mathrm{C}\right)$ 
Table.5 Total flavanoid content (mg CE/g) of dehydrated broccoli florets during the storage intervals

\begin{tabular}{|c|c|c|c|c|}
\hline 0 DAS & $50^{0} \mathrm{C} / \mathrm{B}_{1}$ & $55^{0} \mathrm{C} / \mathrm{B}_{2}$ & $60^{0} \mathrm{C} / \mathrm{B}_{3}$ & Mean A \\
\hline $\mathbf{A}_{1}-\mathbf{A}_{7}$ & 1.26 & 1.13 & 1.02 & - \\
\hline Mean B & - & - & - & \\
\hline 30 DAS & $\mathbf{B}_{1}$ & $\mathbf{B}_{2}$ & $\mathbf{B}_{3}$ & Mean A \\
\hline $\mathbf{T}_{1} / \mathbf{A}_{1}$ & 0.82 & 0.63 & 0.58 & 0.68 \\
\hline $\mathbf{T}_{2} / \mathbf{A}_{2}$ & 0.88 & 0.72 & 0.67 & 0.76 \\
\hline $\mathbf{T}_{\mathbf{3}} / \mathbf{A}_{3}$ & 1.01 & 0.83 & 0.81 & 0.88 \\
\hline $\mathbf{T}_{4} / \mathbf{A}_{4}$ & 0.74 & 0.57 & 0.51 & 0.61 \\
\hline $\mathbf{T}_{5} / \mathbf{A}_{5}$ & 0.94 & 0.77 & 0.72 & 0.81 \\
\hline $\mathrm{T}_{6} / \mathrm{A}_{6}$ & 1.08 & 0.91 & 0.84 & 0.95 \\
\hline $\mathbf{T}_{7} / \mathbf{A}_{7}$ & 0.64 & 0.51 & 0.45 & 0.53 \\
\hline \multirow[t]{5}{*}{ Mean B } & 0.87 & 0.71 & 0.66 & \\
\hline & Factors & C.D. & SE(d) & SE(m) \\
\hline & Factor(A) & 0.010 & 0.005 & 0.003 \\
\hline & Factor(B) & 0.006 & 0.003 & 0.002 \\
\hline & Factor $(\mathrm{AxB})$ & 0.017 & 0.008 & 0.006 \\
\hline 45 DAS & $50^{0} \mathrm{C} / \mathrm{B}_{1}$ & $55^{0} \mathrm{C} / \mathrm{B}_{2}$ & $60^{0} \mathrm{C} / \mathrm{B}_{3}$ & Mean A \\
\hline $\mathbf{T}_{1} / \mathbf{A}_{1}$ & 0.64 & 0.57 & 0.52 & 0.57 \\
\hline $\mathbf{T}_{2} / \mathbf{A}_{2}$ & 0.72 & 0.66 & 0.61 & 0.66 \\
\hline $\mathbf{T}_{3} / \mathbf{A}_{3}$ & 0.84 & 0.79 & 0.69 & 0.77 \\
\hline $\mathbf{T}_{4} / \mathbf{A}_{4}$ & 0.55 & 0.51 & 0.45 & 0.50 \\
\hline $\mathbf{T}_{5} / \mathbf{A}_{5}$ & 0.78 & 0.71 & 0.65 & 0.71 \\
\hline$T_{6} / A_{6}$ & 0.93 & 0.84 & 0.75 & 0.84 \\
\hline $\mathbf{T}_{7} / \mathbf{A}_{7}$ & 0.44 & 0.38 & 0.32 & 0.38 \\
\hline \multirow[t]{5}{*}{ Mean B } & 0.70 & 0.64 & 0.57 & \\
\hline & Factors & C.D. & SE(d) & SE(m) \\
\hline & Factor(A) & 0.011 & 0.006 & 0.004 \\
\hline & Factor(B) & 0.007 & 0.004 & 0.003 \\
\hline & Factor $(\mathrm{AxB})$ & 0.020 & 0.010 & 0.007 \\
\hline 60 DAS & $50^{0} \mathrm{C} / \mathrm{B}_{1}$ & $55^{0} \mathrm{C} / \mathrm{B}_{2}$ & $60^{0} \mathrm{C} / \mathrm{B}_{3}$ & Mean A \\
\hline $\mathbf{T}_{1} / \mathbf{A}_{1}$ & 0.19 & 0.30 & 0.16 & 0.22 \\
\hline $\mathbf{T}_{2} / \mathbf{A}_{2}$ & 0.28 & 0.35 & 0.24 & 0.29 \\
\hline $\mathbf{T}_{\mathbf{3}} / \mathbf{A}_{\mathbf{3}}$ & 0.38 & 0.48 & 0.33 & 0.40 \\
\hline $\mathbf{T}_{4} / \mathbf{A}_{4}$ & 0.15 & 0.26 & 0.09 & 0.17 \\
\hline $\mathbf{T}_{5} / \mathbf{A}_{5}$ & 0.32 & 0.42 & 0.27 & 0.34 \\
\hline $\mathrm{T}_{6} / \mathrm{A}_{6}$ & 0.48 & 0.53 & 0.44 & 0.48 \\
\hline $\mathbf{T}_{7} / \mathbf{A}_{7}$ & 0.11 & 0.15 & 0.06 & 0.11 \\
\hline \multirow[t]{5}{*}{ Mean B } & 0.27 & 0.36 & 0.23 & \\
\hline & Factors & C.D. & SE(d) & SE(m) \\
\hline & Factor(A) & 0.009 & 0.004 & 0.003 \\
\hline & Factor(B) & 0.006 & 0.003 & 0.002 \\
\hline & Factor $(\mathrm{AxB})$ & 0.016 & 0.008 & 0.005 \\
\hline
\end{tabular}

A(1-7): Treatments $\left\{\mathbf{A 1}\left(\mathrm{T}_{1}\right)-\right.$ Citric acid $0.2 \%+4$ min blanching + water, $\mathbf{A 2}\left(\mathrm{T}_{2}\right)-$ Citric acid $0.2 \%+4 \mathrm{~min}$ blanching $+\mathrm{K}_{2} \mathrm{~S}_{2} \mathrm{O}_{5} 0.1 \%, \mathbf{A 3}\left(\mathrm{T}_{3}\right)-$ Citric acid $0.2 \%+4$ min blanching $+\mathrm{Na}_{2} \mathrm{~S}_{2} \mathrm{O}_{5} 0.1 \%, \mathbf{A 4}\left(\mathrm{T}_{4}\right)-\mathrm{CaCl}_{2} 0.2 \%+4$ min blanching + water, $\mathbf{A 5}\left(\mathrm{T}_{5}\right)-\mathrm{CaCl}_{2} 0.2 \%+4$ min blanching $+\mathrm{K}_{2} \mathrm{~S}_{2} \mathrm{O}_{5} 0.1 \%, \mathbf{A 6}\left(\mathrm{T}_{6}\right)-\mathrm{CaCl}_{2} 0.2 \%+4 \min$ blanching $+\mathrm{Na}_{2} \mathrm{~S}_{2} \mathrm{O}_{5} 0.1 \%, \mathbf{A} 7\left(\mathrm{~T}_{7}\right)-$ water +4 min blanching + water $\}, \mathrm{B}(1-3)$ : Temperatures $\left(\mathbf{B} 1-50^{\circ} \mathrm{C}, \mathbf{B} 2-\right.$ $\left.55^{\circ} \mathrm{C}, \mathrm{B3}-60^{0} \mathrm{C}\right)$ 
Table.6 Antioxidant activity (percent inhibition of DPPH) of dehydrated broccoli florets during the storage intervals

\begin{tabular}{|c|c|c|c|c|}
\hline 0 DAS & $50^{0} \mathrm{C} / \mathrm{B}_{1}$ & $55^{0} \mathrm{C} / \mathrm{B}_{2}$ & $60^{0} \mathrm{C} / \mathrm{B}_{3}$ & Mean A \\
\hline $\mathbf{A}_{1}-\mathbf{A}_{7}$ & 38.46 & 33.50 & 32.46 & - \\
\hline Mean B & - & - & - & \\
\hline 30 DAS & $\mathrm{B}_{1}$ & $\mathbf{B}_{2}$ & $\mathbf{B}_{3}$ & Mean A \\
\hline $\mathbf{T}_{1} / \mathbf{A}_{1}$ & 27.83 & 24.47 & 23.17 & 25.16 \\
\hline $\mathbf{T}_{2} / \mathbf{A}_{2}$ & 29.20 & 26.20 & 23.80 & 26.40 \\
\hline $\mathbf{T}_{3} / \mathbf{A}_{3}$ & 32.37 & 31.10 & 27.37 & 30.28 \\
\hline $\mathbf{T}_{4} / \mathbf{A}_{4}$ & 27.10 & 22.57 & 20.57 & 23.41 \\
\hline $\mathbf{T}_{5} / \mathbf{A}_{5}$ & 30.60 & 26.70 & 24.50 & 27.27 \\
\hline $\mathbf{T}_{6} / \mathrm{A}_{6}$ & 35.30 & 31.73 & 28.50 & 31.84 \\
\hline $\mathbf{T}_{7} / \mathbf{A}_{7}$ & 23.20 & 21.37 & 19.50 & 21.36 \\
\hline \multirow[t]{5}{*}{ Mean B } & 29.37 & 26.31 & 23.91 & \\
\hline & Factors & C.D. & SE(d) & $\mathbf{S E}(\mathbf{m})$ \\
\hline & Factor(A) & 0.204 & 0.101 & 0.071 \\
\hline & Factor(B) & 0.134 & 0.066 & 0.047 \\
\hline & Factor AxB & 0.354 & 0.175 & 0.123 \\
\hline 45 DAS & $\mathbf{5 0}^{0} \mathrm{C} / \mathbf{B}_{1}$ & $55^{0} \mathrm{C} / \mathrm{B}_{2}$ & $60^{0} \mathrm{C} / \mathrm{B}_{3}$ & Mean A \\
\hline $\mathbf{T}_{1} / \mathbf{A}_{1}$ & 24.07 & 22.90 & 18.10 & 21.69 \\
\hline $\mathbf{T}_{2} / \mathbf{A}_{2}$ & 24.80 & 24.70 & 18.80 & 22.77 \\
\hline $\mathbf{T}_{3} / \mathbf{A}_{3}$ & 26.63 & 25.33 & 20.63 & 24.20 \\
\hline $\mathbf{T}_{4} / \mathbf{A}_{4}$ & 23.37 & 22.17 & 16.20 & 20.58 \\
\hline $\mathbf{T}_{5} / \mathbf{A}_{5}$ & 25.70 & 24.57 & 19.20 & 23.16 \\
\hline$T_{6} / A_{6}$ & 31.23 & 30.30 & 25.53 & 29.02 \\
\hline $\mathbf{T}_{7} / \mathbf{A}_{7}$ & 20.13 & 18.57 & 13.67 & 17.46 \\
\hline \multirow[t]{5}{*}{ Mean B } & 25.13 & 24.08 & 18.88 & \\
\hline & Factors & C.D. & SE(d) & SE(m) \\
\hline & Factor(A) & 0.156 & 0.077 & 0.054 \\
\hline & Factor(B) & 0.102 & 0.05 & 0.036 \\
\hline & Factor AxB & 0.27 & 0.133 & 0.094 \\
\hline 60 DAS & $50^{0} \mathrm{C} / \mathrm{B}_{1}$ & $55^{0} \mathrm{C} / \mathrm{B}_{2}$ & $60^{0} \mathrm{C} / \mathrm{B}_{3}$ & Mean A \\
\hline $\mathbf{T}_{1} / \mathbf{A}_{1}$ & 17.33 & 17.63 & 10.20 & 15.06 \\
\hline $\mathbf{T}_{2} / \mathbf{A}_{2}$ & 18.17 & 21.17 & 10.80 & 16.71 \\
\hline $\mathbf{T}_{\mathbf{3}} / \mathbf{A}_{\mathbf{3}}$ & 19.27 & 22.57 & 14.63 & 18.82 \\
\hline $\mathbf{T}_{4} / \mathbf{A}_{4}$ & 15.30 & 16.47 & 8.50 & 13.42 \\
\hline $\mathbf{T}_{5} / \mathbf{A}_{5}$ & 18.70 & 21.83 & 11.43 & 17.32 \\
\hline $\mathbf{T}_{6} / \mathbf{A}_{6}$ & 23.73 & 24.00 & 18.53 & 22.09 \\
\hline $\mathbf{T}_{7} / \mathbf{A}_{7}$ & 11.23 & 11.90 & 6.53 & 9.89 \\
\hline \multirow[t]{5}{*}{ Mean B } & 17.68 & 19.37 & 11.52 & \\
\hline & Factors & C.D. & SE(d) & $\mathbf{S E}(\mathbf{m})$ \\
\hline & Factor(A) & 0.203 & 0.1 & 0.071 \\
\hline & Factor(B) & 0.133 & 0.066 & 0.046 \\
\hline & Factor AxB & 0.351 & 0.173 & 0.123 \\
\hline
\end{tabular}

A(1-7): Treatments $\left\{\mathbf{A 1}\left(\mathrm{T}_{1}\right)-\right.$ Citric acid $0.2 \%+4$ min blanching + water, $\mathbf{A 2}\left(\mathrm{T}_{2}\right)-$ Citric acid $0.2 \%+4 \mathrm{~min}$ blanching $+\mathrm{K}_{2} \mathrm{~S}_{2} \mathrm{O}_{5} 0.1 \%, \mathbf{A 3}\left(\mathrm{T}_{3}\right)-$ Citric acid $0.2 \%+4$ min blanching $+\mathrm{Na}_{2} \mathrm{~S}_{2} \mathrm{O}_{5} 0.1 \%, \mathbf{A 4}\left(\mathrm{T}_{4}\right)-\mathrm{CaCl}_{2} 0.2 \%+4$ min blanching + water, $\mathbf{A 5}\left(\mathrm{T}_{5}\right)-\mathrm{CaCl}_{2} 0.2 \%+4$ min blanching $+\mathrm{K}_{2} \mathrm{~S}_{2} \mathrm{O}_{5} 0.1 \%, \mathbf{A 6}\left(\mathrm{T}_{6}\right)-\mathrm{CaCl}_{2} 0.2 \%+4 \min$ blanching $+\mathrm{Na}_{2} \mathrm{~S}_{2} \mathrm{O}_{5} 0.1 \%, \mathbf{A} 7\left(\mathrm{~T}_{7}\right)-$ water +4 min blanching + water $\}, \mathrm{B}(1-3)$ : Temperatures $\left(\mathbf{B} 1-50^{\circ} \mathrm{C}, \mathbf{B} 2-\right.$ $\left.55^{\circ} \mathrm{C}, \mathrm{B3}-60^{\circ} \mathrm{C}\right)$ 


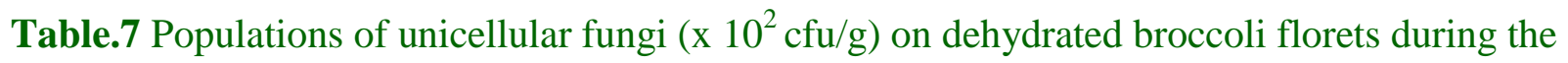
storage intervals

\begin{tabular}{|c|c|c|c|c|}
\hline 0 DAS & $50^{0} \mathrm{C} / \mathrm{B}_{1}$ & $55^{0} \mathrm{C} / \mathrm{B}_{2}$ & $60^{0} \mathrm{C} / \mathrm{B}_{3}$ & Mean A \\
\hline A1-A7 & 2.00 & 2.00 & 1.33 & - \\
\hline Mean B & - & - & - & \\
\hline 30 days & B1 & B2 & B3 & Mean A \\
\hline $\mathbf{T}_{1} / \mathbf{A}_{1}$ & 3.00 & 2.00 & 2.00 & 2.33 \\
\hline $\mathbf{T}_{2} / \mathbf{A}_{2}$ & 2.67 & 1.67 & 1.67 & 2.00 \\
\hline $\mathbf{T}_{3} / \mathbf{A}_{3}$ & 2.00 & 2.00 & 1.67 & 1.89 \\
\hline $\mathbf{T}_{4} / \mathbf{A}_{4}$ & 3.00 & 2.67 & 2.33 & 2.67 \\
\hline $\mathbf{T}_{5} / \mathbf{A}_{5}$ & 2.00 & 1.67 & 1.67 & 1.78 \\
\hline$T_{6} / A_{6}$ & 2.00 & 2.00 & 1.67 & 1.89 \\
\hline $\mathbf{T}_{7} / \mathbf{A}_{7}$ & 3.67 & 3.00 & 2.67 & 3.11 \\
\hline \multirow[t]{5}{*}{ Mean B } & 2.62 & 2.14 & 1.95 & \\
\hline & Factors & C.D. & SE(d) & $\mathbf{S E}(\mathbf{m})$ \\
\hline & Factor(A) & 0.399 & 0.197 & 0.139 \\
\hline & Factor(B) & 0.261 & 0.129 & 0.091 \\
\hline & Factor(A X B) & N/A & 0.341 & 0.241 \\
\hline 45 days & $50^{0} \mathrm{C} / \mathrm{B}_{1}$ & $55^{0} \mathrm{C} / \mathrm{B}_{2}$ & $60^{0} \mathrm{C} / \mathrm{B}_{3}$ & Mean A \\
\hline$T_{1} / A_{1}$ & 3.67 & 2.67 & 2.67 & 3.00 \\
\hline $\mathbf{T}_{2} / \mathbf{A}_{2}$ & 3.67 & 2.33 & 2.33 & 2.78 \\
\hline $\mathbf{T}_{3} / \mathbf{A}_{3}$ & 2.67 & 2.00 & 2.00 & 2.22 \\
\hline $\mathbf{T}_{4} / \mathbf{A}_{4}$ & 3.67 & 3.00 & 2.67 & 3.11 \\
\hline $\mathbf{T}_{5} / \mathbf{A}_{5}$ & 3.00 & 2.33 & 2.00 & 2.44 \\
\hline$T_{6} / A_{6}$ & 1.67 & 1.67 & 1.67 & 1.67 \\
\hline $\mathbf{T}_{7} / \mathbf{A}_{7}$ & 4.00 & 3.67 & 3.00 & 3.56 \\
\hline \multirow[t]{5}{*}{ Mean B } & 3.19 & 2.52 & 2.33 & \\
\hline & Factors & C.D. & SE(d) & $\mathbf{S E}(\mathbf{m})$ \\
\hline & Factor(A) & 0.45 & 0.222 & 0.157 \\
\hline & Factor(B) & 0.295 & 0.145 & 0.103 \\
\hline & Factor(A X B) & N/A & 0.385 & 0.272 \\
\hline 60 days & $50^{\circ} \mathrm{C} / \mathrm{B}_{1}$ & $55^{0} \mathrm{C} / \mathrm{B}_{2}$ & $60^{0} \mathrm{C} / \mathrm{B}_{3}$ & Mean A \\
\hline $\mathbf{T}_{1} / \mathbf{A}_{1}$ & 6.33 & 3.67 & 3.00 & 4.33 \\
\hline $\mathbf{T}_{2} / \mathbf{A}_{2}$ & 4.67 & 3.00 & 2.33 & 3.33 \\
\hline $\mathbf{T}_{\mathbf{3}} / \mathbf{A}_{\mathbf{3}}$ & 3.00 & 2.67 & 2.00 & 2.56 \\
\hline $\mathbf{T}_{4} / \mathbf{A}_{4}$ & 7.00 & 4.33 & 3.33 & 4.89 \\
\hline $\mathbf{T}_{5} / \mathbf{A}_{5}$ & 3.67 & 2.67 & 2.33 & 2.89 \\
\hline $\mathbf{T}_{6} / \mathbf{A}_{6}$ & 2.67 & 2.00 & 2.00 & 2.22 \\
\hline $\mathbf{T}_{7} / \mathbf{A}_{7}$ & 8.67 & 5.67 & 4.00 & 6.11 \\
\hline \multirow[t]{5}{*}{ Mean B } & 5.14 & 3.43 & 2.71 & \\
\hline & Factors & C.D. & SE(d) & $\mathbf{S E}(\mathbf{m})$ \\
\hline & Factor(A) & 0.434 & 0.214 & 0.151 \\
\hline & Factor(B) & 0.284 & 0.140 & 0.099 \\
\hline & Factor(A X B) & 0.751 & 0.371 & 0.262 \\
\hline
\end{tabular}

A(1-7): Treatments $\left\{\mathbf{A 1}\left(\mathrm{T}_{1}\right)-\right.$ Citric acid $0.2 \%+4$ min blanching + water, $\mathbf{A 2}\left(\mathrm{T}_{2}\right)-$ Citric acid $0.2 \%+4 \mathrm{~min}$ blanching $+\mathrm{K}_{2} \mathrm{~S}_{2} \mathrm{O}_{5} 0.1 \%, \mathbf{A 3}\left(\mathrm{T}_{3}\right)-$ Citric acid $0.2 \%+4$ min blanching $+\mathrm{Na}_{2} \mathrm{~S}_{2} \mathrm{O}_{5} 0.1 \%, \mathbf{A 4}\left(\mathrm{T}_{4}\right)-\mathrm{CaCl}_{2} 0.2 \%+4$ min blanching + water, $\mathbf{A 5}\left(\mathrm{T}_{5}\right)-\mathrm{CaCl}_{2} 0.2 \%+4$ min blanching $+\mathrm{K}_{2} \mathrm{~S}_{2} \mathrm{O}_{5} 0.1 \%, \mathbf{A 6}\left(\mathrm{T}_{6}\right)-\mathrm{CaCl}_{2} 0.2 \%+4 \min$ blanching $+\mathrm{Na}_{2} \mathrm{~S}_{2} \mathrm{O}_{5} 0.1 \%, \mathbf{A} 7\left(\mathrm{~T}_{7}\right)-$ water +4 min blanching + water $\}, \mathrm{B}(1-3)$ : Temperatures $\left(\mathbf{B} 1-50^{\circ} \mathrm{C}, \mathbf{B} 2-\right.$ $\left.55^{\circ} \mathrm{C}, \mathrm{B3}-60^{\circ} \mathrm{C}\right)$ 
Table.8 Populations of filamentous fungi $\left(\mathrm{x} 10^{2} \mathrm{cfu} / \mathrm{g}\right)$ on dehydrated broccoli florets during the storage intervals

\begin{tabular}{|c|c|c|c|c|}
\hline 0 DAS & $50^{0} \mathrm{C} / \mathrm{B}_{1}$ & $55^{0} \mathrm{C} / \mathrm{B}_{2}$ & $60^{0} \mathrm{C} / \mathrm{B}_{3}$ & Mean A \\
\hline A1-A7 & 0.66 & 0.66 & 0.66 & - \\
\hline Mean B & - & - & - & \\
\hline 30 days & B1 & B2 & B3 & Mean A \\
\hline$T_{1} / A_{1}$ & 1.33 & 1.33 & 1.00 & 1.22 \\
\hline $\mathbf{T}_{2} / \mathbf{A}_{2}$ & 1.33 & 1.33 & 1.00 & 1.22 \\
\hline $\mathbf{T}_{\mathbf{3}} / \mathbf{A}_{3}$ & 1.33 & 1.33 & 0.67 & 1.11 \\
\hline $\mathbf{T}_{4} / \mathbf{A}_{4}$ & 1.33 & 1.67 & 1.33 & 1.44 \\
\hline $\mathbf{T}_{5} / \mathbf{A}_{5}$ & 1.33 & 1.00 & 0.67 & 1.00 \\
\hline$T_{6} / A_{6}$ & 1.33 & 1.33 & 0.67 & 1.11 \\
\hline $\mathbf{T}_{7} / \mathbf{A}_{7}$ & 2.00 & 1.67 & 1.67 & 1.78 \\
\hline \multirow[t]{5}{*}{ Mean B } & 1.429 & 1.381 & 1.00 & \\
\hline & Factors & C.D. & SE(d) & $\mathbf{S E}(\mathbf{m})$ \\
\hline & Factor(A) & N/A & 0.245 & 0.173 \\
\hline & Factor(B) & 0.325 & 0.16 & 0.113 \\
\hline & Factor(A X B) & N/A & 0.424 & 0.300 \\
\hline 45 days & ${ }^{50} 0^{0} \mathrm{C} / \mathrm{B}_{1}$ & $55^{0} \mathrm{C} / \mathrm{B}_{2}$ & $60^{0} \mathrm{C} / \mathrm{B}_{3}$ & Mean A \\
\hline $\mathrm{T}_{1} / \mathrm{A}_{1}$ & 1.67 & 1.67 & 1.33 & 1.56 \\
\hline $\mathbf{T}_{2} / \mathbf{A}_{2}$ & 1.67 & 1.67 & 1.33 & 1.56 \\
\hline $\mathbf{T}_{\mathbf{3}} / \mathbf{A}_{\mathbf{3}}$ & 1.33 & 1.33 & 1.00 & 1.22 \\
\hline $\mathbf{T}_{4} / \mathbf{A}_{4}$ & 1.67 & 2.00 & 1.67 & 1.78 \\
\hline $\mathbf{T}_{5} / \mathbf{A}_{5}$ & 1.67 & 1.33 & 1.00 & 1.33 \\
\hline $\mathbf{T}_{6} / \mathbf{A}_{6}$ & 1.33 & 1.00 & 0.67 & 1.00 \\
\hline $\mathbf{T}_{7} / \mathbf{A}_{7}$ & 2.33 & 2.00 & 1.67 & 2.00 \\
\hline \multirow[t]{5}{*}{ Mean B } & 1.67 & 1.57 & 1.24 & \\
\hline & Factors & C.D. & SE(d) & $\mathbf{S E}(\mathbf{m})$ \\
\hline & Factor(A) & 0.481 & 0.238 & 0.168 \\
\hline & Factor(B) & 0.315 & 0.156 & 0.110 \\
\hline & Factor(A X B) & N/A & 0.411 & 0.291 \\
\hline 60 days & $5^{0} \mathrm{C} / \mathrm{B}_{1}$ & $55^{0} \mathrm{C} / \mathrm{B}_{2}$ & $60^{0} \mathrm{C} / \mathrm{B}_{3}$ & Mean A \\
\hline $\mathbf{T}_{1} / \mathbf{A}_{1}$ & 2.00 & 2.00 & 1.67 & 1.89 \\
\hline $\mathbf{T}_{2} / \mathbf{A}_{2}$ & 2.00 & 1.67 & 1.33 & 1.67 \\
\hline $\mathbf{T}_{\mathbf{3}} / \mathbf{A}_{\mathbf{3}}$ & 1.67 & 1.67 & 1.00 & 1.44 \\
\hline $\mathbf{T}_{4} / \mathbf{A}_{4}$ & 2.33 & 2.33 & 2.00 & 2.22 \\
\hline $\mathbf{T}_{5} / \mathbf{A}_{5}$ & 2.00 & 1.67 & 1.33 & 1.67 \\
\hline$T_{6} / A_{6}$ & 1.33 & 1.00 & 0.67 & 1.00 \\
\hline $\mathbf{T}_{7} / \mathbf{A}_{7}$ & 2.67 & 2.67 & 2.33 & 2.56 \\
\hline \multirow[t]{5}{*}{ Mean B } & 2.00 & 1.86 & 1.48 & \\
\hline & Factors & C.D. & SE(d) & $\mathbf{S E}(\mathbf{m})$ \\
\hline & Factor(A) & 0.450 & 0.222 & 0.157 \\
\hline & Factor(B) & 0.295 & 0.145 & 0.103 \\
\hline & Factor(A X B) & N/A & 0.385 & 0.272 \\
\hline
\end{tabular}

A(1-7): Treatments $\left\{\mathbf{A 1}\left(\mathrm{T}_{1}\right)-\right.$ Citric acid $0.2 \%+4$ min blanching + water, $\mathbf{A 2}\left(\mathrm{T}_{2}\right)-$ Citric acid $0.2 \%+4$ min blanching $+\mathrm{K}_{2} \mathrm{~S}_{2} \mathrm{O}_{5} 0.1 \%, \mathbf{A 3}\left(\mathrm{T}_{3}\right)-$ Citric acid $0.2 \%+4$ min blanching $+\mathrm{Na}_{2} \mathrm{~S}_{2} \mathrm{O}_{5} 0.1 \%, \mathbf{A 4}\left(\mathrm{T}_{4}\right)-\mathrm{CaCl}_{2} 0.2 \%+4$ min blanching + water, $\mathbf{A 5}\left(\mathrm{T}_{5}\right)-\mathrm{CaCl}_{2} 0.2 \%+4$ min blanching $+\mathrm{K}_{2} \mathrm{~S}_{2} \mathrm{O}_{5} 0.1 \%, \mathbf{A 6}\left(\mathrm{T}_{6}\right)-\mathrm{CaCl}_{2} 0.2 \%+4$ min blanching $+\mathrm{Na}_{2} \mathrm{~S}_{2} \mathrm{O}_{5} 0.1 \%, \mathbf{A} 7\left(\mathrm{~T}_{7}\right)-$ water +4 min blanching + water $\}, \mathrm{B}(1-3)$ : Temperatures $\left(\mathbf{B 1}-50^{\circ} \mathrm{C}\right.$, B2$\left.55^{\circ} \mathrm{C}, \mathbf{B 3}-60^{\circ} \mathrm{C}\right)$ 
Fig.1 Moisture content (dry weight basis) of broccoli florets at variable temperature during the process of dehydration

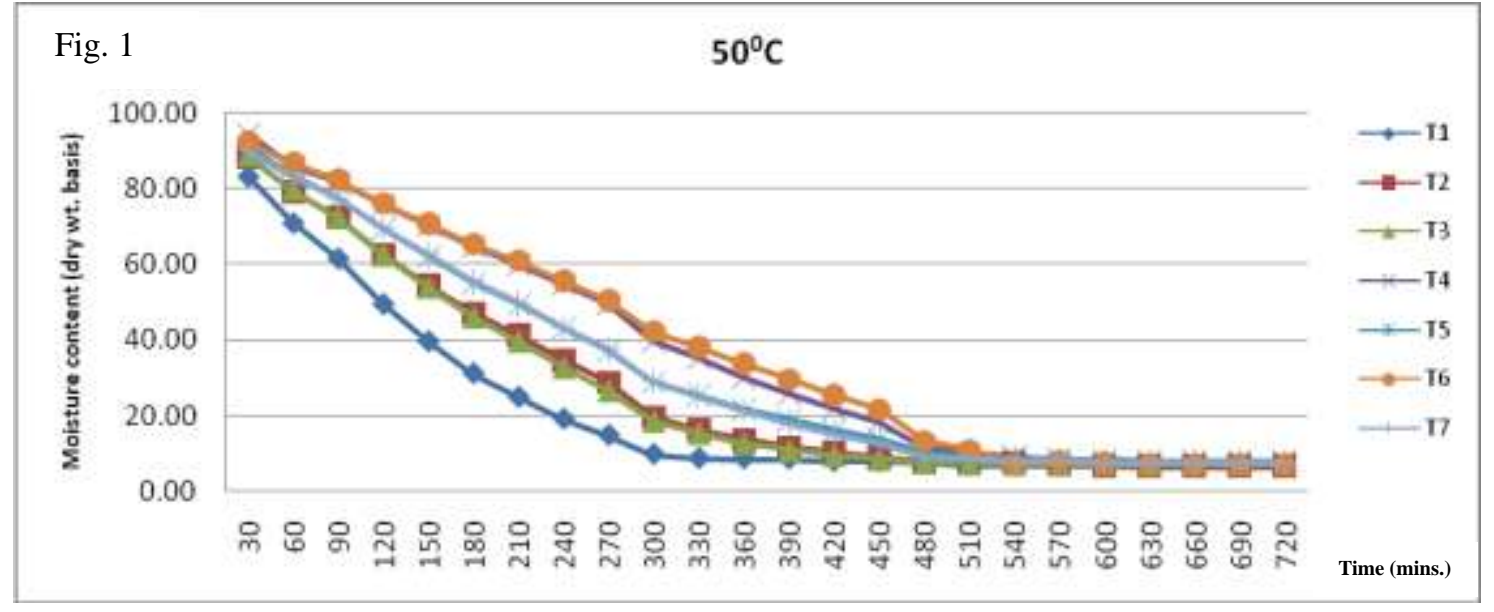

Fig.2 Moisture content (dry weight basis) of broccoli florets at variable temperature during the process of dehydration

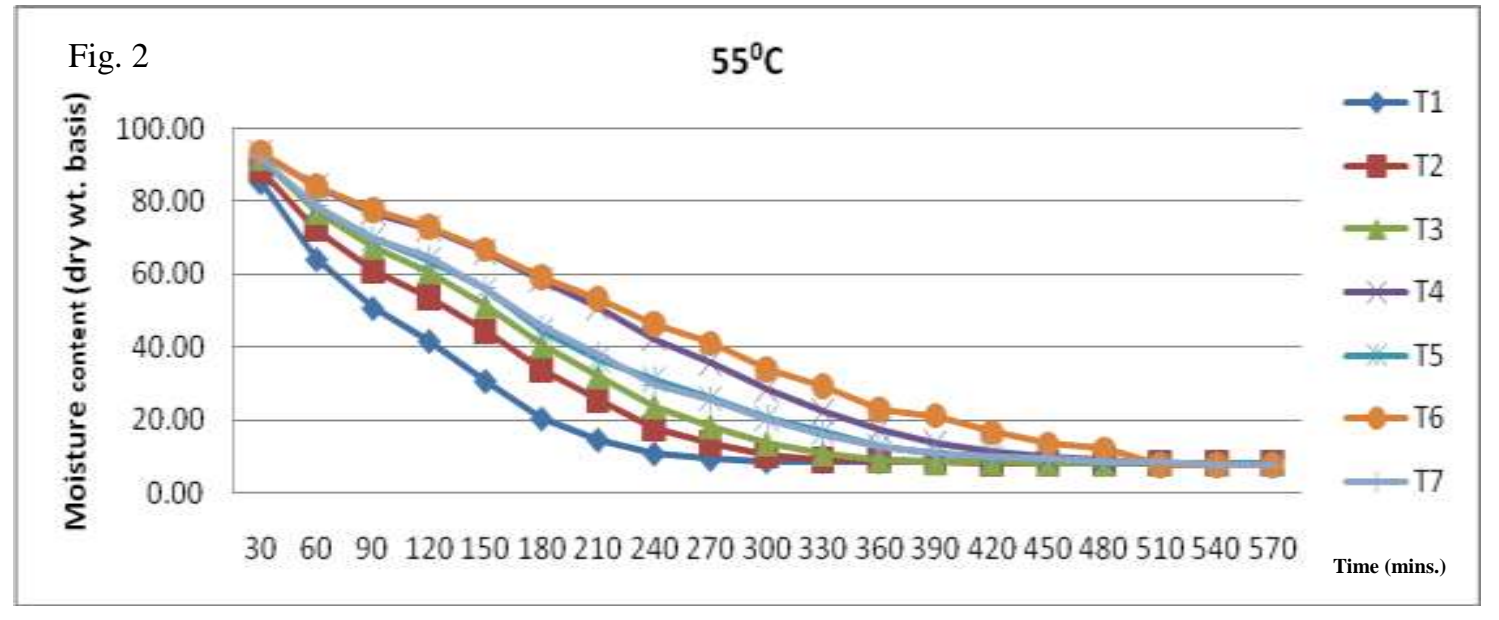

Fig.3 Moisture content (dry weight basis) of broccoli florets at variable temperature during the process of dehydration

Fig. 3

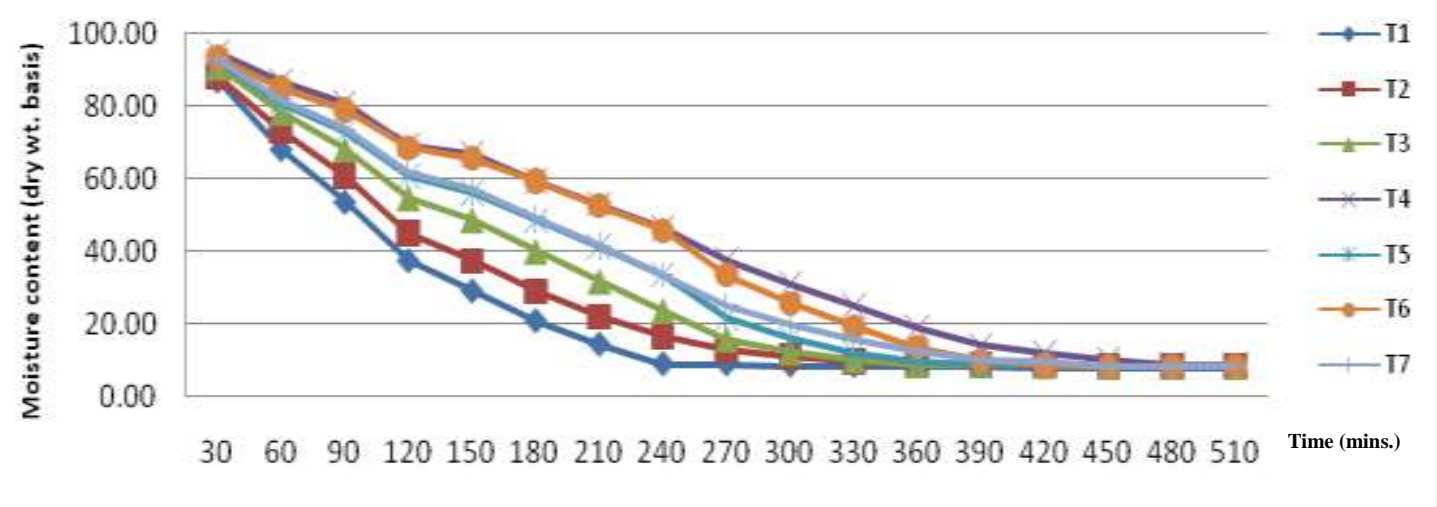


Das and Dhua (2019) reported similar observation for dehydrated banana inflorescence on several attributes.

The study showed that at 0 days of storage various physical and chemical attributes for dehydrated broccoli florets were at their maximum for treatments dehydrated at $50^{\circ} \mathrm{C}$ followed by treatments dehydrated at $55^{\circ} \mathrm{C}$ and treatments dehydrated at $60^{\circ} \mathrm{C}$. However later during the study, concentration of various biochemical parameters decreased for treatments dehydrated at $50^{\circ} \mathrm{C}$ and treatments dehydrated at $60^{\circ} \mathrm{C}$ respectively. At first 30 days of storage maximum concentration of total chlorophyll, phenols, flavanoids and antioxidants were seen for treatments dehydrated at $50^{\circ} \mathrm{C}$ followed by treatments dehydrated at $55^{\circ} \mathrm{C}$ and lastly for treatments dehydrated at $60^{\circ} \mathrm{C}$. But at 45 days of storage various attributes for different treatments dehydrated at $50^{\circ} \mathrm{C}$ decreased as compared to treatments dehydrated at $55^{\circ} \mathrm{C}$. Similar trend was seen at 60 days of storage. The biochemical parameters and other physical attributes for treatments dehydrated at $60^{\circ} \mathrm{C}$ were always low. The fungal populations for treatments dehydrated at $60^{\circ} \mathrm{C}$ was lesser than treatments dehydrated at $55^{\circ} \mathrm{C}$ and treatments dehydrated at $50^{\circ} \mathrm{C}$ but the population count of treatments dehydrated at $55^{\circ} \mathrm{C}$ were very much similar to that of the treatments dehydrated at $60^{\circ} \mathrm{C}$. So lastly it was concluded that the temperature of $55^{\circ} \mathrm{C}$ for dehydration of broccoli florets and pre drying treatment of initial immersion with $0.2 \%$ of calcium chloride, 4 minutes of hot water blanching and final immersion with $0.1 \%$ of sodium metabisulfite was most effective which helped in retaining the physical and biochemical properties throughout the storage period.

\section{Acknowledgement}

The first author of this experiment acknowledges INSPIRE Fellowship
Programme under Department of Science and Technology, Ministry of Science and Technology, New Delhi for providing monetary aid during the experiment.

\section{References}

A.O.A.C. 2000. Official Methods of Analysis. 17th Ed. Association of Official Analytical Chemists, Horwitz, USA.

Allen, O.N. 1953. Experiments in Soil Bacteriology. Burgess Co., Minneapolis, Minn. pp. 69-70.

Brand-Williams, W., Cuvelier, M.E. and Berset, C. 1995. Use of a free radical method to evaluate antioxidant activity. LWT Food Sci Tech., 28: 25-30.

Das, A and Dhua, R.S. 2019. Standardization of drying techniques to develop ready to cook banana inflorescence. Int.J.Curr.Microbiol.App.Sci. $\quad 8(03)$ : 1523-1536.

Gullett, N.P., Amin, A.R.R., Bayraktar, S., Pezzuto, J.M. and Shin, D.M. 2010. Cancer prevention with natural compounds. Semin Oncol., 37: 258-281.

Hatamipour, M.S., Kazemi, H.H., Nooralivand, A and Nozarpoor, A. 2007. Drying Characteristics of six varieties of sweet potaoes in different dryers. Food Bioprod. Process., 85(3): 171-177.

Izumi, A. and Watada, A.E. 1994. Calcium treatments affect storage quality of shredded carrots. J. Food Sci., 59: 106109.

Kingsly, A.R.P., Singh, R., Goyal, R.K. and Singh, D.B. 2007. Thin-layer drying behaviour of organically produced tomato. Am. J. Food Technol., 2: 71-78.

Kordylas, J.M. 1990. Processing and Preservation of Tropical and Subtropical Foods. Macmillan Publishers Ltd. London.

Kunzek, H., Kabbert, R. and Gloyna, D. 1999. Aspects of material science in food processing: changes in plant cell walls of fruits and vegetables. 208(4): 233-250.

McEvily, A.J., Iyengar, R. and Otwell, W.S. 
1992. Inhibition of enzymatic browning in foods and beverages. Critical Reviews in Food Science and Nutrition., 32(3): 253-273.

Munyaka, A.W., Oey, I., Van Loey, A. and Hendrickx, M. 2010. Application of thermal inactivation of enzymes during vitamin $\mathrm{C}$ analysis to study the influence of acidification, crushing and blanching on vitamin $\mathrm{C}$ stability in Broccoli (Brassica oleracea L var. italica). Food Chem., 120(2): 591-598.

Ngangom, R., Reang, P. and Das, A. (2019). Study on development of suitable ways for preparation and preservation of dehydrated pomegranate arils. International Journal of Chemical Studies., 7(5): 1034-1038.

Owureku, M., Amponesh, J., Saalia, F., Alfaro, L., Luis, A. and Sathivel, S. 2014. Effect of pretreatment on physicochemical quality characteristic of a dried tomato. Afr. J. Food Sci., 8(5): 253-259.

Picchioni, G. A. 1994. Membrane lipid metabolism, cell permeability and ultra structural changes in lightly processed carrot. J. Food Sci., 59: 601-605.

Platel, K. and Srinivasan, K. 1997. Plant foods in the management of diabetes mellitus: vegetables as potential hypoglycaemic agents. Nahrung., 41: 68-74.

Rangana, S. 2003. Handbook Of Analysis and Quality Control for Fruit and Vegetables Products $2^{\text {nd }}$ ed., Tata McGraw Hill.pp., 12-16.

Rosen, J. C. and Kader, A.A. 1989. Postharvest physiology and quality maintenance of sliced pear and strawberry fruits. J. Food Sci., 54: 656-659.

Sheoran, O.P., Tonk, D.S., Kaushik, L.S., Hasija, R.C. and Pannu, R.S. 1998. Statistical Software Package for Agricultural Research Worker. Recent Advances in information theory, Statistics and Computer Applications by D.S. Hooda and R.C. Hasija, Department of Mathematics Statistics, CCS HAU, Hisar (139-143).

Shipley, B. and $\mathrm{Vu}$, T. T. 2002. Dry matter content as a measure of dry matter concentration in plants and their parts. New Phytol. 153, 359-364.

Singleton, V.L., Orthofer, R. and LamuelaRaventos, R.M. 1999. Analysis of total phenols and other oxidation substrates and antioxidants by means of FolinCiocalteau reagent. Methods Enzymol., 299: 152-178.

Vámos-Vigyázó, L. 1995. Prevention of Enzymatic Browning in Fruits and Vegetables, Enzymatic Browning and Its Prevention. American Chemical Society, pp. 49-62.

Waldron, K.W., Parker, M.L. and Smith, A.C. 2003. Plant cell wall and food quality: A review. J. Sci. Food Technol., 2:109-10.

Zhishen, J., Mengcheng, T. and Jianming, W. 1999. The determination of flavonoid contents in mulberry and their scavenging effects on superoxide radicals. Food Chem., 64: 555-59.

\section{How to cite this article:}

Ankan Das and Dhua, R. S. 2019. Study for Determination of Suitable Pre treatment Combination and Dehydration Temperature for Broccoli Florets. Int.J.Curr.Microbiol.App.Sci. 8(10): 727-741. doi: https://doi.org/10.20546/ijcmas.2019.810.084 